23 October 2020

\title{
Neural Organoids and the Precautionary Principle
}

Commentary on: Henry T. Greely, Human Brain Surrogates Research: The Onrushing Ethical Dilemma, American Journal of Bioethics, 2021.

\section{Jonathan Birch}

Centre for Philosophy of Natural and Social Science, London School of Economics and Political Science.

j.birch2@,1se.ac.uk

ORCID: https://orcid.org/0000-0001-7517-4759

\section{Heather Browning}

Centre for Philosophy of Natural and Social Science, London School of Economics and Political Science.

ORCID: http://orcid.org/0000-0003-1554-7052 


\begin{abstract}
It is a familiar idea that we should "apply the precautionary principle" when designing animal welfare regulations: we should not allow our uncertainty about the sentience of some animals to delay the adoption of proportionate measures to protect those animals from severe welfare threats. The same general idea should be applied to neural organoid research. If any of a set of neurological "warning signs" is found, proportionate measures should be taken to mitigate welfare risks. A measure likely to be proportionate is to bring the organoids in question within the scope of regulation that already exists for scientific research on sentient animals (such as, in a UK context, the "ASPA" regulatory framework).
\end{abstract}


Human neural organoid research is advancing rapidly. As Greely notes in his target article, this progress presents an “onrushing ethical dilemma”. We can't rule out the possibility that sufficiently sophisticated organoids are, or will soon be, sentient: capable of having feelings with a positive or negative quality, such as feelings of pain or pleasure. If they are sentient, then there are moral limits on what we can do to them, and regulation is urgently needed to prevent research overstepping those limits.

In other contexts, it is a familiar idea that we should "apply the precautionary principle" when designing animal welfare regulations. We should not allow our uncertainty about the sentience of some animals to delay the adoption of proportionate measures to protect those animals from severe welfare threats. For example, we should not allow our uncertainty about the sentience of octopods to prevent us from regulating scientific research on octopods. We have written elsewhere about the questions of detail that arise when we apply precautionary thinking to the case of invertebrate welfare (Birch 2017; Browning 2017).

The same general idea is attractive in the context of neural organoids. We should not allow our uncertainty about their sentience to block the adoption of proportionate measures to safeguard their welfare. But what would it be to apply a precautionary principle to neural organoid research? Our aim here is to start a discussion with some initial proposals.

It will help to start with an approach I think we should not take. Koplin and Savulescu (2020) have argued that, because "we can be reasonably confident that a brain organoid lacks even a rudimentary form of consciousness until it resembles the brain of a fetus at 20 weeks' development" (Koplin and Savulescu, p. 762), no additional regulation should be required for research on organoids that are equivalent to a foetal brain at 20 weeks or less. Although 
Koplin and Savulescu take this to be erring "on the side of generosity", we do not share their confidence about these cases. Given the obvious ethical constraints on research on human foetuses, our knowledge of when sentience begins remains subject to severe uncertainty. Derbyshire and Bockmann (2020) have suggested that, to err on the side of caution, we should regard foetuses as potentially sentient from 12 weeks, since this is the time of the first known projections from the thalamus into the cortical subplate.

Yet this does not mean that we should regard organoid research as unproblematic as long as the organoids are equivalent to a foetal brain of 12 weeks or less. It is misguided in general, we suggest, to use highly uncertain estimates about human foetuses as a guide to the ethics of neural organoid research. Instead, we should expect information to flow in the opposite direction: we should look directly for markers of sentience in organoids, draw conclusions about how small an organoid can be and yet still display these markers, and then use this evidence to formulate better policies regarding human foetuses.

What sort of markers can we look for? Here we face a serious problem: in the case of nonhuman animals, the most compelling markers of sentience tend to be behavioural. For example, if an animal learns to self-administer anaesthetics or analgesics (such as opioids) in response to injury, that is some evidence that it is having an aversive experience. Animal welfare experts have formulated lists of such markers (e.g. Sneddon et al. 2014). Yet none of these behavioural markers of sentience are likely to be present in an organoid, because organoids are cut off (either partially or totally) from the sources of perceptual input and motor output that are available to a developed organism. We need to rely on non-behavioural markers. 
Yet no theory about the neural correlates of conscious experience (NCCs) in humans is uncontroversial, and different groups of researchers emphasize different brain regions and processes. Some argue that conscious experience depends on local activity in a particular area of the cortex, perhaps the "posterior hot zone" (Koch et al. 2016), while others argue that conscious experience depends on global ignition of many cortical regions (Mashour et al. 2020). Some highlight the role of thalamocortical connections (Aru et al. 2019), while others argue that midbrain regions such as the superior colliculus may be sufficient by themselves for conscious experience (Merker 2007). While views that focus on the cortex and the thalamus are considered more mainstream than Merker's midbrain-centred view, none can be decisively ruled out.

What to do in this state of severe uncertainty? Here is a proposal: if an organoid contains structures or mechanisms that any serious and credible theory of the human NCCs posits to be sufficient for conscious experience, we should take proportionate measures to regulate research on that organoid. In practice, this sets the evidential bar for taking precautions at an intentionally low level. In particular: if Merker's midbrain theory is accepted as serious and credible (as we think it should be), then an organoid in which functioning midbrain mechanisms can be discerned, of the type regarded as sufficient for sentience by that theory, has cleared the bar. This remains the case even though Merker's theory has few proponents and even though the evidence for it is fairly weak (Birch 2020). Precautionary thinking requires us to take seriously theories of consciousness that can't be ruled out on the basis of current evidence, even if they don't command strong positive evidential support.

If organoids are developed with a discernible midbrain, thalamus or cortical subplate, what response would be proportionate? The most obvious precaution is that, in these 
circumstances, the organoids should be brought within the regulatory frameworks that currently exist in many countries for scientific research on sentient animals. The UK already has a rigorous framework based on the Animals (Scientific Procedures) Act 1986 ("ASPA"), requiring ethical review, a careful weighing of harms and benefits, and evidence that scientists have duly considered the imperative to reduce, refine, and replace. We suggest it would be a proportionate response to bring any organoid displaying neurological "warning signs" of sentience within the scope of ASPA. As we understand it, the technology is not there yet, but, given the slow pace of regulatory change in relation to scientific progress, it would be wise to prepare the necessary regulatory changes now.

Some may object: if we regulate research on potentially sentient organoids, and introduce an imperative to reduce, refine and replace, isn't it possible that we will miss out on major medical advances? But the same counterargument arises for non-human animals, where it is usually regarded as unpersuasive. Regulation is not about stopping research. Once a regulatory framework is in place, harms can be weighed against benefits, and a case can be made for the value to society of those benefits. The aim would be to block the gratuitous use of potentially sentient organoids when simpler model systems that are less likely to be sentient could be used instead.

Before concluding, we will comment briefly on the other "human brain surrogates" discussed by Greely. Precautionary thinking of the type just outlined may well apply to research on ex vivo human brains, if we reach a stage where whole brain regions are being maintained after death. For gene-edited animals and chimaeras, the issues are somewhat different. Precautionary reasoning may still be useful, but in a different way. 
In these cases, sentience is not usually at issue — a modified lab rat, for example, can be presumed sentient. There is, however, a knowledge gap surrounding the welfare needs of substantially modified or novel animals (Browning 2018). In making decisions about the best treatment for animals, we need information about which conditions will enhance or detract from their welfare. Typically, this information is gathered either through studying wild counterparts, or else through testing the effects of different housing and husbandry conditions. This requires either a population of wild relatives or an existing captive population of the same (or relevantly similar) animals. For novel animal types, such as geneedited animals and chimaeras, we lack such populations. In particular, animals with humanlike brain traits are likely to have different, potentially more demanding welfare needs in comparison with their unedited counterparts. For example, an environment that might be stimulating for an unedited animal might be a source of agonising boredom for an animal cognitively enhanced with human cortical tissue. This is likely to lead to poor welfare outcomes. This knowledge gap leads to a second precautionary suggestion: when evaluating the harms and benefits of research on human brain surrogates, we should recognise our own ignorance regarding their welfare needs, and take into account the risk of unforeseen harm that results from this ignorance.

\section{Acknowledgements}

This research is part of a project that has received funding from the European Research Council (ERC) under the European Union's Horizon 2020 research and innovation programme, Grant No. 851145. 
References

Aru, J., Suzuki, M., Rutiku, R., Larkum, M. E. and Bachmann, T. 2019. Coupling the state and contents of consciousness. Frontiers in Systems Neuroscience. 13:43. doi: $10.3389 /$ fnsys. 2019.00043

Birch, J. 2017. Animal sentience and the precautionary principle. Animal Sentience 2(16):1. Retrieved from https://animalstudiesrepository.org/animsent/vol2/iss16/1/

Birch, J. 2020. The search for invertebrate consciousness. Noûs. doi: 10.1111/nous.12351

Browning. H. 2017. Anecdotes can be evidence too. Animal Sentience 2(16):13. Retrieved from https://www.wellbeingintlstudiesrepository.org/animsent/vol2/iss16/13/

Browning, H. 2018. Won't somebody please think of the mammoths? De-extinction and animal welfare. Journal of Agricultural and Environmental Ethics. 31:785-803

Derbyshire, S. W. G. and Bockmann, J. C. 2020. Reconsidering fetal pain. Journal of Medical Ethics 46:3-6. doi:10.1136/medethics-2019-105701

Koch, C., Massimini, M., Boly, M. and Tononi, G. 2016. Neural correlates of consciousness: Progress and problems. Nature Reviews Neuroscience 17:307-322. https://doi.org/10.1038/nrn.2016.22

Koplin, J. J. and Savulescu, J. 2020. Moral limits of brain organoid research. Journal of Law, Medicine \& Ethics 47: 760-767. doi: 10.1177/1073110519897789

Mashour, G. A., Roelfsema, P., Changeux, J. P., and Dehaene, S. 2020. Conscious processing and the global neuronal workspace hypothesis. Neuron 105:776-798.

Merker, B. 2007. Consciousness without a cerebral cortex: A challenge for neuroscience and medicine. Behavioral and Brain Sciences 30:63-134.

Sneddon, L. U., Elwood, R. W., Adamo, S. A. and Leach, M. C. 2014. Defining and assessing animal pain. Animal Behaviour 97:201-212. http://dx.doi.org/10.1016/j.anbehav.2014.09.007 